41. Mena I, Court J, Fuenzalida S, Papavasiliou PS, Cotzias GC. Modification of chronic manganese poisoning: treatment with L-dopa or 5-OH tryptophane. N Engl J Med 1970;282:5-10.

42. Tanner CM, Ottman R, Goldman SM, et al. Parkinson disease in twins: an etiologic study. JAMA 1999;281:341-346.

43. Brooks DJ, Ibanez V, Sawle GV, et al. Differing patterns of striatal 18F-dopa uptake in Parkinson's disease, multiple system atrophy, and progressive supranuclear palsy. Ann Neurol 1990;28:547-555.

44. Wolters EC, Huang CC, Clark C, et al. Positron emission tomography in manganese intoxication. Ann Neurol 1989;26: 647-651.
45. Eriksson H, Tedroff J, Thuomas KA, et al. Manganese induced brain lesions in Macaca fascicularis as revealed by positron emission tomography and magnetic resonance imaging. Arch Toxicol 1992;66:403-407.

46. Mena I, Court J, Fuenzalida S, Papavasilou PS, Cotzias GC. Chronic manganese poisoning: clinical picture and manganese turnover. Neurology 1967;17:128-136.

47. Shinotoh H, Snow BJ, Hewitt KA, et al. MRI and PET studies of manganese-intoxicated monkeys. Neurology 1995;45:1199 1204.

48. Mirowitz SA, Westrich TJ, Hirgsch JD. Hyperintense basal ganglia on T1-weighted MR images in patients receiving parenteral nutrition. Radiology 1991;181:117-120.

\title{
Relationship of substantia nigra echogenicity and motor function in elderly subjects
}

\author{
Daniela Berg, MD; Christiane Siefker; Petra Ruprecht-Dörfler, MD; and Georg Becker, MD
}

\begin{abstract}
Article abstract-Background: Patients with Parkinson's disease (PD) exhibit an increased echogenicity of the substantia nigra (SN) on transcranial sonography. Some healthy adults with the same echo characteristics showed a reduced ${ }^{18}$ fluorodopa uptake on PET, indicating a subclinical alteration of the nigrostriatal system. Objectives: To determine whether the sonographic phenotype of hyperechogenic SN has any relevance for motor function in elderly subjects and whether an increased echogenicity of the SN is associated with an impaired motor function. Method: In a populationbased, cross-sectional study, 93 subjects older then 60 years without history of extrapyramidal disorder underwent sonographic and neurologic examinations, with a quantitative motor assessment. Results: Elderly healthy subjects without prediagnosed extrapyramidal disorder but with SN hyperechogenicity had more frequent and more severe parkinsonian symptoms and a slower finger tapping than those with a regular echogenicity of the SN ( $p<0.05, U$ test). Conclusion: With increasing age, subjects with SN hyperechogenicity develop a more substantial slowing of movements than subjects without this echo pattern, stressing the functional relevance of this sonographic finding. The authors speculate that hyperechogenicity of the SN may be detected by transcranial sonography early in life and may serve as a risk marker for nigral injury, although only a minority of these subjects will develop the full clinical picture of PD.
\end{abstract}

NEUROLOGY 2001;56:13-17

The majority of patients with Parkinson's disease (PD) exhibit an increased echogenicity of the substantia nigra (SN) on transcranial sonography (TCS). ${ }^{1}$ The mechanisms resulting in an increased echogenicity of the SN are still unclear. The same echo pattern of the SN has been identified in approximately $9 \%$ of healthy adults, with only a slight increase of the prevalence rate with age. ${ }^{2}$ Recent ${ }^{18}$ fluorodopa ([$\left.{ }^{18} \mathrm{~F}\right]$-dopa) PET studies disclosed a marked decrease in the accumulation of $\left[{ }^{18} \mathrm{~F}\right]$-dopa in the striatum in some healthy subjects, with this increased SN echogenicity indicating a functional impairment of the nigrostriatal system. ${ }^{2}$ According to these findings, we speculate that ultrasound may identify an important susceptibility marker for PD, and that the mechanisms causing this increase in
SN echogenicity make nigral neurons more vulnerable to other noxious factors such as endo- or exotoxines. ${ }^{3,4}$

This study was designed to test the hypothesis that the subclinical alteration of nigral neurons detected by TCS might become symptomatic during life, and whether subjects with SN hyperechogenicity tend to develop clinical symptoms of the nigrostriatal system and, eventually, PD. The prevalence rate of $\mathrm{PD}$ is far below the rate of SN hyperechogenicity. ${ }^{5}$ Therefore, at most, $5 \%$ of subjects with SN hyperechogenicity will develop typical PD. Nevertheless, one may ask whether subjects with SN hyperechogenicity more often exhibit motor slowing and stiffness with increasing age compared with subjects without this ultrasound finding. These subjects 


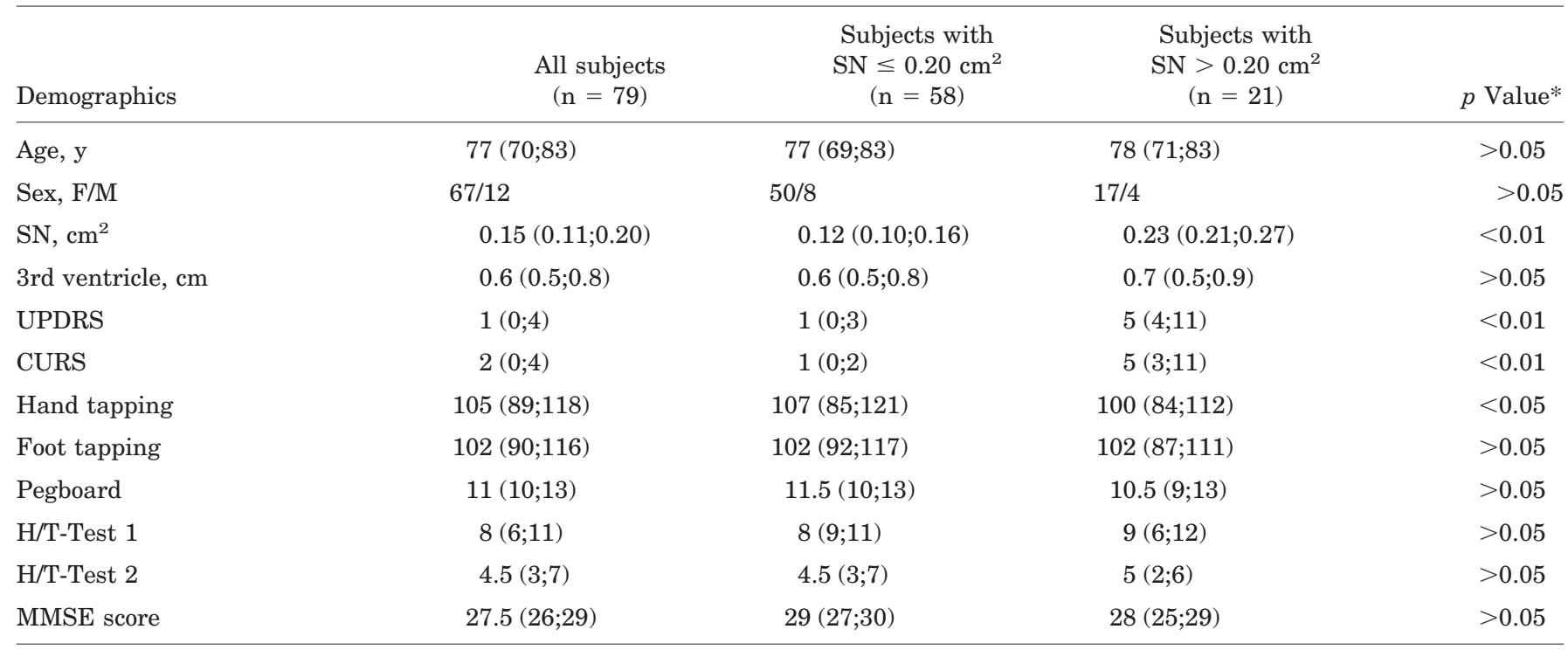

Values expressed as median value (25th percentile; 75 th percentile).

* Mann-Whitney $U$ test.

$\mathrm{SN}=$ substantia nigra; UPDRS $=$ Unified PD Rating Scale; CURS = Columbia University Rating Scale; MMSE = Mini-Mental State Examination.

might acquire typical PD if they live long enough. Hence, we sought to reveal the functional relevance of increased echogenicity of the $\mathrm{SN}$ and to determine whether subjects older than 60 years show a relationship between motor function and echo pattern of the SN, and whether individuals with SN hyperechogenicity more often exhibit clinical symptoms attributable to nigrostriatal system alteration.

Subjects and methods. We performed a cross-sectional, population-based study that included 93 individuals from senior citizens' homes and church groups for active elderly people of the city of Würzburg, Germany. All individuals gave informed consent according to the Declaration of Helsinki. Inclusion criteria were as follows: age above 60 years; no prediagnosed extrapyramidal disorders, especially no PD; and no medical disorder severely affecting the motility of the subject. All subjects underwent a thorough neurologic examination and comprehensive motor and neuropsychological testing. Neurologic examination included the Columbia University Rating Scale (CURS) ${ }^{6}$ and the motor examination part (part III) of the Unified PD Rating Scale (UPDRS). ${ }^{7}$ Individuals exhibiting only one of the cardinal symptoms of PD (bradykinesia, tremor, rigor) with minor expression were classified to exhibit subtle signs of nigrostriatal dysfunction. The diagnosis of PD was established in accordance with the UK PD brain bank criteria. ${ }^{8}$

To quantify motor function, a pegboard examination and a series of finger- and foot-tapping tests were administered. ${ }^{2,9,10}$ Each subject was asked to tap a clip and a pedal as fast as possible with the first two fingers of each hand and both feet separately twice for 16 seconds. Results were recorded by computer, and a median value for each hand and foot was calculated. For the pegboard examination, small metal sticks had to be set into little holes on a board twice with each hand separately for 30 seconds. A median value of correctly set sticks for each hand was calculated.

Neuropsychological assessment was performed using the Mini-Mental State Examination (MMSE) ${ }^{11}$ and the H/T-Word Test. ${ }^{12,13}$ The H/T-Word Test is a neuropsychological assessment for executive functions in which subjects are asked to produce as many different words as possible within 2 minutes beginning alternately with the letters "H" and "T." This test is known to be abnormal in early stages of PD .2,14

The subjects' medical and social history was carefully taken, including the assessment of epidemiologic data such as area of birth, residential background, work, exposure to toxic agents (particularly herbicides, pesticides, and mercurycontaining solutions), use of alcohol or smoking, as well as family and medical history. Some of these factors are known to be associated with the development of PD ${ }^{4,5}$

For TCS examination, a color-coded, phased array ultrasound system equipped with a $2.5 \mathrm{MHz}$ transducer was used (Elegra, Siemens, Erlangen, Germany). The examination was performed through a preauricular acoustic bone window with a penetration depth of $16 \mathrm{~cm}$ and a dynamic range of $45 \mathrm{~dB}$. The $\mathrm{SN}$ was identified within the butterflyshaped structure of the mesencephalic brainstem as clearly as possible, scanning from both temporal bone windows, and quantified by encircling and measuring the area of hyperechogenic signals in the SN region as previously described. ${ }^{2} 30$ subjects were reassessed by a second ultrasound physician, who was blinded to the results of the first physician, to determine the reproducibility of the measurement. The widths of the third ventricle and the frontal horns of the lateral ventricles were measured on a standardized diencephalic scanning plane. ${ }^{15}$

All examinations were performed on the same day by independent investigators blinded to the results of all 
other examinations. Only the assessment of epidemiologic data and the neurologic investigation was done by the same investigator.

Statistics. Descriptive statistics are given as median with lower (25th percentile) and upper (75th percentile) quartile. Correlation analysis was performed by Spearman rank correlation. Intergroup comparison was performed by the Mann-Whitney $U$ test.

Results. TCS examination was performed in 79 of the 93 subjects; 14 subjects had no appropriate temporal acoustic bone windows and were therefore excluded from further analysis. In the remaining 79 subjects ( 67 women, 12 men, median age 77 [70 to 83] years) the mesencephalic brainstem was adequately displayed by TCS, allowing measurements of the area of hyperechogenic signals at the SN. Median area of SN echogenicity was $0.14 \mathrm{~cm}^{2}(0.11$ to 0.19 $\left.\mathrm{cm}^{2}\right)$ for the right and $0.15 \mathrm{~cm}^{2}\left(0.11\right.$ to $\left.0.20 \mathrm{~cm}^{2}\right)$ for the left side (table). Eleven subjects (13.9\%) had a distinctly hyperechogenic SN, with an echogenic signal exceeding $0.25 \mathrm{~cm}^{2}$ on one or both sides. The cut off of $0.25 \mathrm{~cm}^{2}$ was used in our recent studies because it corresponded to the 90th percentile of hyperechogenic signal extent at the SN in the healthy population. ${ }^{2}$ In this group of elderly subjects, we found no correlation between signal extension at the SN and age ( $r=0.17, p>0.05$; Spearman rank correlation) nor was there a dependence on gender $(p>0.05, U$ test). Sonographic measurements of signal extension proved adequately reproducible considering the smallness of the structure measured (1st examination: $0.15[11,18]$ $\mathrm{cm}^{2}$, 2nd examination: 0.15 [12,18] $\mathrm{cm}^{2} ; r=0.49, p<0.01$; Spearman rank correlation).

Neurologic examination revealed a mild to moderate increase of muscle tone predominating on one side of the body in 26 subjects, tremor at rest in four subjects, and action tremor in five subjects. Significant bradykinesia with lateralization to the right side was noticed in five patients and to the left side in seven patients. Eight subjects showed a reduced swinging of one arm during walking. Twelve subjects were unable to get up out of a chair without upper extremity support. The median frequencies for finger tapping differed for the right hand (110 [93;123]/ $32 \mathrm{~s})$ and the left hand $(100[89 ; 110] / 32 \mathrm{~s})$. Frequencies for foot tapping were $103(92 ; 116) / 32$ seconds for the right foot and $100(87 ; 115) / 32$ seconds for the left foot. The decline in tapping frequency with age was significant $(r=0.22, p<$ 0.05 ; Spearman rank correlation). The average CURS and UPDRS-III scores were $2(0 ; 4)$ and $1(0 ; 4)$. In eight subjects, CURS and UPDRS-III scores were above 10. Two individuals fulfilled the diagnostic criteria of for PD, considering the UK PD brain bank criteria. ${ }^{8}$ Cognitive function was found to be impaired in 14 subjects with MMSE scores of 25 or lower. The median MMSE score was $27.5(26 ; 29) ; \mathrm{H} / \mathrm{T}$ scores were $8(6 ; 11)$ after the first minute and $4(3 ; 7)$ after the second minute. We identified a negative correlation of age and MMSE or H/T scores $(p<0.02$; Spearman rank correlation), but no correlation of age and CURS or UPDRS scores ( $p$ $>0.05$; Spearman rank correlation).

Thirty-three subjects had been living in rural environments, 46 mainly in urban environments. Three patients reported a positive family history of PD and six reported a history of essential tremor. Seven patients had been exposed to environmental toxins, and one had been on neuroleptic drugs. In this small group, no association between

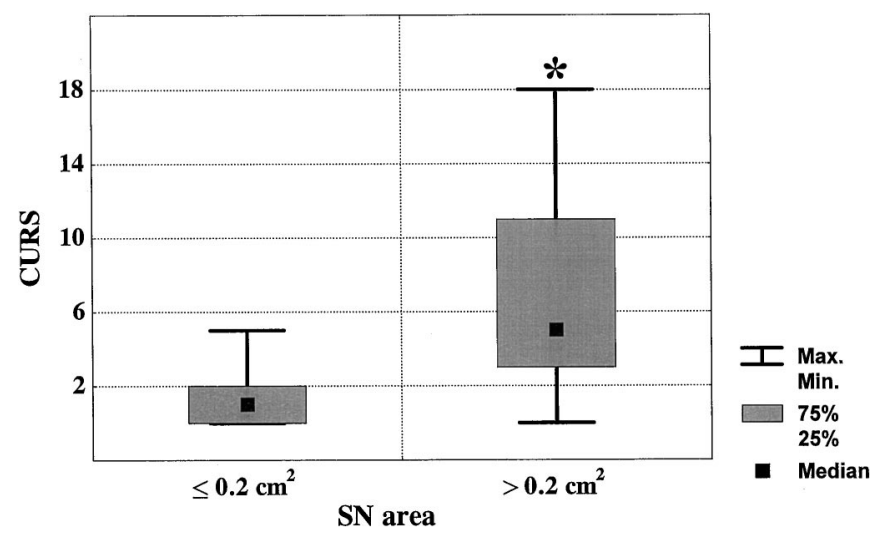

Figure 1. Elderly subjects with more extended hyperechogenic areas at the substantia nigra (SN) showed more severe motor deficits as determined by the Columbia University Rating Scale (CURS). Difference in CURS was significant $(\mathrm{p}<0.001 ; \mathrm{U}$ test).

these epidemiologic factors and the assessments of motor function could be identified ( $p>0.05, U$ test).

The comparison of ultrasound findings with motor assessment documented a correlation of hyperechogenic areas at the SN on both sides and the CURS or UPDRS scores $(r=0.47, p<0.001$ and $r=0.45, p<0.001$; Spearman rank correlation). Those with an extension of hyperechogenic signal beyond the 75th percentile $(>0.20$ $\left.\mathrm{cm}^{2}\right)$ showed higher CURS and UPDRS scores $(p<0.01 ; U$ test; figure 1). In parallel, subjects with CURS scores above 10 had larger echogenic areas at the SN than those with lower CURS scores ( $p<0.01 ; U$ test). In addition, we found a negative correlation of hyperechogenic signal extension and frequency of the finger tapping of the contralateral hand $(r=-0.19, p=0.02$; Spearman rank correlation). Finger tapping in individuals with SN echogenicity $\leq 0.2 \mathrm{~cm}^{2}$ compared to those with an area of SN echogenicity $>0.2 \mathrm{~cm}^{2}$ differed also $(p=0.04$; $U$ test; figure 2). Differences remained when controlling for the age of the subjects ( $p=0.02$; analysis of variance). Foot tapping or pegboard examinations did not correlate with measurements of SN echogenicity, nor did MMSE or H/T Word Test ( $p>0.05$; Spearman rank correlation). Subjects with a positive family history for PD had on average more extended

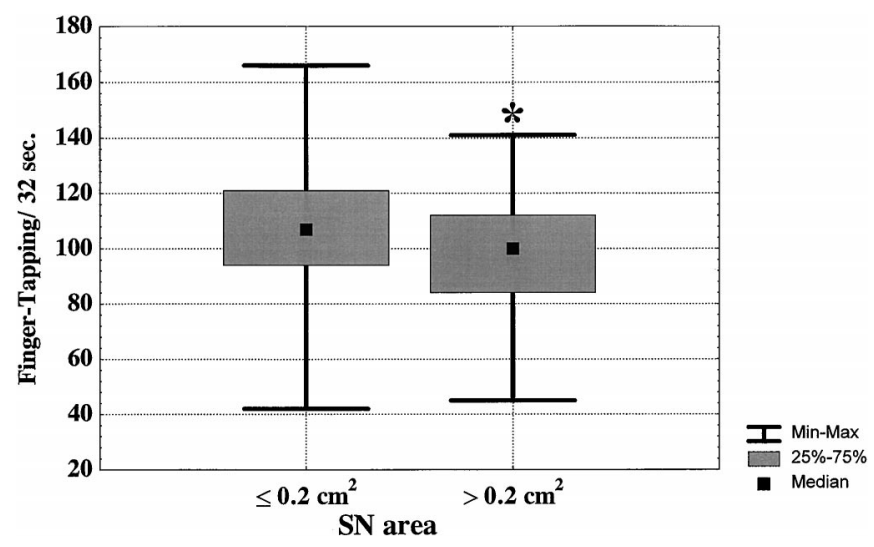

Figure 2. Comparison of finger-tapping frequencies in individuals with area of substantia nigra (SN) echogenicity $>0.2$ and $\leq 0.2 \mathrm{~cm}^{2}$. Comparison was significant ( $\mathrm{p}=0.04$; $\mathrm{U}$ test). 
hyperechogenic signals at the $\mathrm{SN}\left(0.2[0.12 ; 0.28] \mathrm{cm}^{2}\right)$ than those with a negative history $\left(0.15[0.11 ; 0.19] \mathrm{cm}^{2}\right)$. However, differences were not significant. There was no difference in SN echogenicity when patients with positive and negative family history of essential tremor were compared.

The ventricular width was negatively correlated to the cognitive function of the subjects: the larger the third ventricle, the lower the scores of the MMSE and H/T-Word Test $(r=-0.42, p<0.001$ and $r=-0.41, p<0.001$; Spearman rank correlation).

Discussion. This study shows an association between area of SN hyperechogenicity detected by TCS and signs of extrapyramidal deficits, ranging from subtle signs of nigrostriatal dysfunction to symptoms that fulfilled the diagnostic criteria for PD, in subjects without prediagnosed extrapyramidal disorders. Subjects older than 60 years with more extended hyperechogenic signals at the SN showed, on average, more severe motor slowing and more frequent features typical for $\mathrm{PD}$ such as rigor, tremor, or bradykinesia. In the affected subjects, these symptoms had been attributed to normal aging, a misjudgment that is common and results in a significant underestimation of patients suffering from nigrostriatal dysfunction. Results of this study complement previous findings reporting on an injury of the nigrostriatal system in younger individuals with a hyperechogenicity of the SN. ${ }^{2}$ Younger subjects (median age 30 [27;38] years) with a distinctly hyperechogenic SN exhibited a significant decrease of $\left[{ }^{18} \mathrm{~F}\right]$-dopa uptake at the striatum on PET scans, indicating a functional alteration of the nigrostriatal system. Motor examination in these younger subjects was normal, indicating that nigrostriatal injury was subclinical and compensated at this age. Findings of this study, however, document that this may not hold true for elderly individuals as a significant number of subjects with SN hyperechogenicity turn out to be "symptomatic" and show more often and more severe motor retardation or parkinsonian symptoms compared to those lacking this echogenicity pattern.

A previous study revealed that the prevalence rate of subjects displaying hyperechogenicity of the $\mathrm{SN}$ increases only slightly with increasing age indicating that this sonographic marker of nigral vulnerability may develop early in life and may remain almost unchanged during life time. ${ }^{2}$ If this is true, one may speculate that the elderly subjects of our study with SN hyperechogenicity and motor slowing might have had a hyperechogenic SN early in their life, which could have been detected with ultrasound. In support of this hypothesis, follow-up examinations in patients with PD and healthy controls during the last 6 years demonstrated an almost unchanged extension of hyperechogenic signals at the SN. However, prospective longitudinal studies are needed to further validate this hypothesis and to determine whether ultrasound may disclose an important susceptibility marker for nigral injury before the outbreak of neurologic deficits.
The reason for the increased echogenicity of the SN remains unknown. According to recent data from animal experiments ${ }^{16}$ and preliminary postmortem examinations, we speculate that increased echogenicity of the SN reflects a higher tissue iron content. Iron is known to facilitate the generation of superoxide and hydroxyl radicals; this results in oxidative stress, which may end up in irreversible cell injury and death of the cell. ${ }^{17-20}$

Increased echogenicity of the SN is likely to be but one risk factor for nigral injury because not all elderly subjects with SN hyperechogenicity showed signs of motor retardation, and only a minority developed overt PD. Other factors may be necessary to increase cell injury at the $\mathrm{SN}$-notably, environmental factors such as exotoxins. Although we did not detect an association of the development of parkinsonian signs and epidemiologic data assessed in our study, such influences cannot be ruled out, as our study population was rather small. Only a small number of individuals affected by exotoxins and only a few factors known to be associated with PD were assessed.

\section{References}

1. Becker G, Seufert J, Bogdahn U, Reichmann H, Reiners K. Degeneration of substantia nigra in chronic Parkinson's disease visualized by transcranial color-coded real-time sonography. Neurology 1995;45:182-184.

2. Berg D, Becker G, Zeiler B, et al. Vulnerability of the nigrostriatal system as detected by transcranial ultrasound. Neurology 1999;53:1026-1031.

3. Irwin I, Langston JW. Endogenous toxins as potential etiologic agents in Parkinson's disease. In: Ellenberg JH, Koller WC, Langston JW, eds. Etiology of Parkinson's disease. New York, NY: Marcel Dekker Inc, 1995:153-202.

4. Seidler A, Hellenbrand W, Robra B-P, et al. Possible environmental, occupational, and other etiologic factors for Parkinson's disease: a case control study in Germany. Neurology 1996;46:1275-1284.

5. Golbe LI. The genetics of Parkinson's disease: a reconsideration. Neurology 1990;40(suppl 13):7-14.

6. Montgomery GK, Reynolds NC, Warren MR. Quantitative assessment of Parkinson's disease: study of reliability and data reduction with an abbreviated Columbia scale. Clin Neuropharmacol 1985;8:83-92.

7. Fahn S, Elton RL, Members of the UPDRS Development Committee. Unified Parkinson's disease rating scale. In: Fahn S, Marsden CD, Goldstein M, et al., eds. Recent developments in Parkinson's disease II. New York, NY: Macmillan, 1987;153-163.

8. Hughes AJ, Daniel SE, Kilford L, Les AJ. Accuracy of clinical diagnosis and idiopathic Parkinson's disease: a clinicopathological study of 100 cases. J Neurol Neurosurg Psychiatry 1992;55:181-184.

9. Hietanen M, Teravainen H, Tsui JK, et al. The pegboard as a measurement of Parkinsonian deficits. Neurology 1987; 37(suppl):266. Abstract.

10. Vingerhoets FJ, Schulzer M, Calne DB, Snow BJ. Which clinical sign of Parkinson's disease best reflects the nigrostriatal lesion? Ann Neurol 1997;41:58-64.

11. Folstein MF, Folstein SE, McHugh PR. "Mini-Mental State": a practical method for grading the cognitive state of patients for the clinician. J Psychiatr Res 1975;12:189-198.

12. Lezak MD. Neuropsychological assessment, 3rd ed. New York, NY: Oxford University Press, 1995.

13. Zimmermann P, Fimm B. A computerized neuropsychological assessment of attention deficits. Freiburg, Germany: Institute of Psychology, University of Freiburg, 1989. 
14. Lange KW, Paul GM, Naumann M, Gsell W. Dopaminergic effects on cognitive performance in patients with Parkinson's disease. J Neural Transm 1995;46(suppl):423-432.

15. Becker G, Bogdahn U, Straßburg H-M, et al. Identification of ventricular enlargement and estimation of ventricular pressure by transcranial color-coded real-time sonography. J Neuroimaging 1994;4:17-22.

16. Berg D, Grote C, Rausch WD, et al. Iron accumulation in the substantia nigra in rats visualized by ultrasound. Ultrasound Med Biol 1999;25:901-904.

17. Youdim MBH, Ben-Shachar D, Eshel G, Finberg JPM, Riederer
P. The neurotoxicity of iron and nitric oxide. Relevance to the etiology of Parkinson's disease. Adv Neurol 1993;60:259-266.

18. Dexter DT, Sian J, Jenner P, Marsden CD. Implications of alterations in trace element levels in brain in Parkinson's disease and other neurological disorders affecting the basal ganglia. Adv Neurol 1993;60:273-281.

19. Gerlach M, Ben-Shachar D, Riederer P, Youdim MBH. Altered brain metabolism of iron as a cause of neurodegenerative diseases? J Neurochem 1994;63:793-807.

20. Jellinger KA. The role of iron in neurodegeneration. Drugs Aging 1999;14:115-160.

\title{
cME Competency to consent to medical treatment in cognitively impaired patients with Parkinson's disease
}

\author{
Maureen P. Dymek, PhD; Paul Atchison, MD; Lindy Harrell, MD, PhD; and Daniel C. Marson, JD, PhD
}

Article abstract-Objectives: To investigate capacity to consent to medical treatment (competency) in cognitively impaired patients with PD. Background: Although competency has been studied empirically in patients with cortical dementia (AD), no empirical studies have examined competency in patients with PD or other subcortical neurodegenerative disorders. Methods: Patients with PD with cognitive impairment $(\mathrm{n}=20)$ and older controls $(\mathrm{n}=20)$ were compared using a standardized competency measure (Capacity to Consent to Treatment Instrument [CCTI]) and neuropsychological test measures. The CCTI tests competency performance and assigns outcomes (capable, marginally capable, incapable) under four different legal standards (LS). Results: Patients with PD performed below controls on the four LS: capacity to evidence a treatment choice (LS1) $(p<0.03)$, capacity to appreciate consequences of a treatment choice (LS3) $(p<0.03)$, capacity to provide rational reasons for a treatment choice (LS4) $(p<0.0001)$, and capacity to understand the treatment situation and choices (LS5) $(p<0.0001)$. With respect to competency outcomes, patients with PD demonstrated increasing compromise (marginally capable or incapable outcomes) across the four standards: LS1 (25\%), LS3 (45\%), LS4 (55\%), and LS5 (80\%). In the PD group, simple measures of executive function (the Executive Interview) and to a lesser extent memory/orientation (Dementia Rating Scale, Memory subscale) were key predictors of competency performance and outcome on the LS. Conclusions: Cognitively impaired patients with PD are likely to have impaired consent capacity, and are at risk of losing competency over the course of their neurodegenerative illness. Patients with PD have particular difficulty meeting more stringent, clinically relevant competency standards that tap reasoning skills and comprehension of treatment information. Executive dysfunction appears to be a primary neurocognitive mechanism for competency loss in PD.

NEUROLOGY 2001;56:17-24

Between one quarter and one half of patients with $\mathrm{PD}$ are estimated to also have dementia..$^{1-3}$ Cognitive deficits in PD commonly emerge in the areas of higher order attention, memory, spatial skills, and in particular, executive function. ${ }^{4,5}$ Executive function

Additional material related to this article can be found on the Neurology Web site. Go to www.neurology.org and scroll down the Table of Contents for the January 9 issue to find the title link for this article. deficits are among the most prominent cognitive deficits in PD, and may be one of the earliest signs of cognitive deterioration in the disorder.4,6,7 Furthermore, executive function deficits may underlie other cognitive impairments in PD, such as memory, verbal fluency, reasoning, spatial skills, and complex attention..$^{4,5}$

Cognitive deficits presumably contribute to functional declines experienced by patients with $\mathrm{PD}$.

From the Department of Psychiatry (Dr. Dymek), University of Chicago, IL; Department of Neurology (Drs. Atchison, Harrell, and Marson), Alzheimer's Disease Research Center (Drs. Harrell and Marson), and Center for Aging (Drs. Harrell and Marson), University of Alabama at Birmingham; and the Veterans' Administration Medical Center (Dr. Harrell), Birmingham, AL.

Supported in part by an Alzheimer's Disease Center Cores grant (NIH, NIA 1 P30 AG10163-1), an Alzheimer's Disease Program Project grant (NIH, NIA 5 P01 AG06569-05), an Alzheimer's Disease Research Center grant (NIH, NIA 1P50 AG16582-01), and an Alzheimer's Association Pilot Research Grant (PRG 91-122).

Received April 24, 2000. Accepted in final form September 28, 2000.

Address correspondence and reprint requests to Dr. Daniel Marson, Department of Neurology, JT1216, University of Alabama at Birmingham, Birmingham, AL; e-mail: dmarson@uab.edu 


\section{Neurology}

Relationship of substantia nigra echogenicity and motor function in elderly subjects Daniela Berg, Christiane Siefker, Petra Ruprecht-Dörfler, et al.

Neurology 2001;56;13-17

DOI 10.1212/WNL.56.1.13

\section{This information is current as of January 9, 2001}

\section{Updated Information \&} Services

References

Citations

Subspecialty Collections

Permissions \& Licensing

Reprints including high resolution figures, can be found at: http://n.neurology.org/content/56/1/13.full

This article cites 14 articles, 4 of which you can access for free at: http://n.neurology.org/content/56/1/13.full\#ref-list-1

This article has been cited by 11 HighWire-hosted articles: http://n.neurology.org/content/56/1/13.full\#\#otherarticles

This article, along with others on similar topics, appears in the following collection(s):

All Movement Disorders

http://n.neurology.org/cgi/collection/all_movement_disorders

Gait disorders/ataxia

http://n.neurology.org/cgi/collection/gait_disorders_ataxia

Parkinson's disease/Parkinsonism

http://n.neurology.org/cgi/collection/parkinsons_disease_parkinsonism Ultrasound

http://n.neurology.org/cgi/collection/ultrasound

Information about reproducing this article in parts (figures,tables) or in its entirety can be found online at:

http://www.neurology.org/about/about_the_journal\#permissions

Information about ordering reprints can be found online:

http://n.neurology.org/subscribers/advertise

Neurology ${ }^{\circledR}$ is the official journal of the American Academy of Neurology. Published continuously since 1951, it is now a weekly with 48 issues per year. Copyright . All rights reserved. Print ISSN: 0028-3878. Online ISSN: 1526-632X.

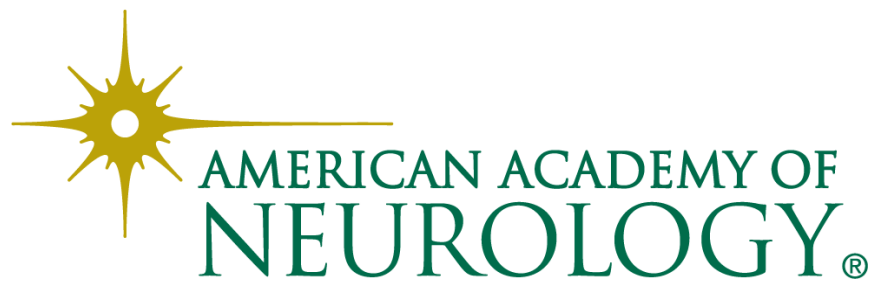

